\title{
Using the Griffiths III and Quartile Charts in Assessing Autism Spectrum Disorder: A Case Study
}

\author{
Jennifer M. Jansen ${ }^{1}$, Elizabeth Green ${ }^{1,2}$, Louise A. Stroud ${ }^{1,2} \&$ Mark B. Watson ${ }^{1}$ \\ ${ }^{1}$ Psycholology Department, Nelson Mandela University, Port Elizabeth, South Africa \\ ${ }^{2}$ Association for Infant and Child Development, Manchester, United Kingdom \\ Correspondance: Jennifer M. Jansen, Psychology Department, Nelson Mandela University, Port Elizabeth, South \\ Africa. E-mail: Jennifer.jansen@mandela.ac.za
}

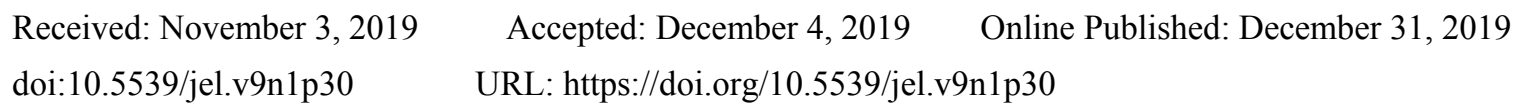

\begin{abstract}
This study illustrates the use of the Griffiths III Scales to futher clarify a diagnosis of Autistic Spectrum Disorder and possible Attention Deficit/Hyperactivity Disorder following a test battery of the Childhood Autism Rating Scale, Conners 3-Parent and Teacher Surveys, and the Goodenough-Harris Draw-a-Person test in a child aged 6 years. The Griffiths III assessment confirmed a pattern of delay above and below DQ50. The child demonstrated difficulty with the constructs: Skills for Learning (including attention, curiosity, processing speed); Ways of Thinking, Memory and Play; all aspects of language and communication; and all underlying constructs of early social and emotional development. The concept of Theory of Mind is used to examine the child's particular difficulties.
\end{abstract}

Keywords: autism spectrum disorder, Griffiths III, developmental assessment, case study, quartile charts

\section{Introduction}

The assessment of atypical young children is a challenging process, particularly as such assessment is not only for diagnostic purposes. The goal of the assessment information is ultimately to stimulate and develop the child's learning path by assisting parents to understand their child's functioning, and teachers to maximise the assessment results in order to utilise the child's strengths and challenges in promoting successful learning at a pace the child is able to cope with. There is no single method of obtaining this information and no generalised intervention strategies. Some health practitioners use a bio-psychosocial approach to assessment, while other health practitioners use a medical model to assess atypical children. Whatever evaluation method is used, it needs to inform the learning process as to what support is needed for the child. The present article reports a case study that illustrates the use of a specific battery of selected assessment measures, together with a theoretical framework, namely Theory of Mind (ToM), to conceptualise a diagnosis with the aim of assisting the client and the family.

\section{Reason for Referral and Sources of Information of Case Study}

$\mathrm{JJ}$ is a 6 years and 4 months old boy that currently attends pre-school, which is a level of schooling that caters for children from the age of five, in order to develop skills needed for successful formal learning. JJ was referred for a psychometric assessment to confirm a provisional diagnosis of Autistic Spectrum Disorder (ASD). Various health care professionals, namely a paediatrician, a speech therapist, an intern psychologist and an audiologist from a state hospital, also requested confirmation of possible Attention Deficit/Hyperactivity Disorder (ADHD). The information on which the assessment is based was obtained through extensive interviews with both parents, working individually with $\mathrm{JJ}$, as well as seeking collateral information on his behaviour from his current pre-school teacher.

\section{Case History}

Salient information reported by the health care professionals and JJ's parents included several behavioural manifestations displayed both at home and during the various medical interventions. Significant delays in reaching key developmental milestones were noted by the parents, who also reported that JJ experienced consistent delays in his communication development, labile moods, self-destructive behaviours and frequent extreme emotions without evidencing a middle ground. JJ has been prescribed Ritalin (for his ADHD symptoms) 
and Risperidone (as a mood-stabiliser). His parents reported that this has a positive effect on his general functioning and that he tends to be calmer after taking the medication.

Despite parental collateral history and reported possible features of ASD by other health care professional, behaviours displayed during the time spent with these professionals were insufficient to make a conclusive diagnosis. In order to reach diagnostic clarity, a decision was made to assess JJ using a psychometric battery. The test battery consisted of the Childhood Autism Rating Scale, $2^{\text {nd }}$ Edition, the Conners 3-Parent and Teacher Surveys, Long Form and the Goodenough-Harris Draw-a-Person test (Conners, 2008; Goodenough, 1963; Schopler, Van Bourgondien, Wellman, \& Love, 2010).

The Childhood Autism Rating Scale (CARS) is a behavioural rating scale used for assessing the presence and severity of symptoms of autism spectrum disorders. It is a clinician-rated assessment used to identify children with ASD, and to determine symptom severity through quantifiable ratings based on direct observation. During the assessment there were certain tasks JJ was unwilling to engage in. Those he did engage indemonstrated him to be a careful and meticulous worker, suggesting that he is likely to be receptive to educational instruction. Although it was possible to maintain his interest by playing with some toys, JJ did not engage in reciprocal play. Generally, he did not initiate conversation and only responded when directly prompted. When interpreting the different categories measured, it was evident that $\mathrm{JJ}$ obtained moderate to severely impaired scores on the 'Adaptation to Change/Restricted Interests' scale as well as the Verbal and Non-verbal communication domains. JJ's overall results on the CARS2-placed him at the $90^{\text {th }}$ percentile of symptom levels compared to individuals with Autism Spectrum Diagnoses.

The Conners 3-Parent and Teacher Surveys, Long Form was included in the assessment battery as both the parents and the teacher reported that $\mathrm{JJ}$ is highly distractible and shows various impulsive behaviours that hinder the learning process. The measure is used to screen for ADHD symptoms and provides information on a range of behavioural and attentional domains. These include inattention, hyperactivity/impulsivity, indicators of problems with learning and executive functioning, levels of defiance/aggression, and peer relations. It further screens for possible symptoms of Conduct Disorder and Oppositional Defiant Disorder. JJ meets the diagnostic criteria for ADHD Combined Presentation (both inattentive and hyperactive/impulsive), Oppositional Defiant Disorder (ODD) and Conduct Disorder (CD).

The Goodenough-Harris Draw-a-Person Intellectual Ability Test (DAP: IQ) is intended to measure a child's developmental and intellectual maturity and was included in the assessment battery. This drawing was scored and rated according to DAP: IQ scoring principles. JJ's drawing produced an IQ equivalent of 95, which places him at the 37th percentile. This implies that 63 percent of children his age would be expected to perform better than him on this measure. His performance can be qualitatively described as average (DAP: IQ, 1963).

These assessments confirmed that JJ meets the diagnostic criteria for Autism Spectrum Disorder [299.00 (F84.00)] as stipulated in the Diagnostic and Statistical Manual of Mental Disorders, Fifth Edition (American Psychiatric Association, 2013). In addition to reaching diagnostic clarity, it is imperative that the assessment process ultimately informs parents and educators in a manner that will guide them as to how to use diagnostic results effectively in order to develop a meaningful support programme for the child assessed. From a treatment perspective, knowing the diagnostic label alone is hardly enough information to decide on the best course of action to take. Furthermore, individuals diagnosed with ASD have variable symptoms, and therefore merely providing a diagnostic label does not encapsulate the nuances of the individual being assessed. For example, a person with a diagnosis of ASD might have significant sensory issues, while another might struggle more with language. These two individuals will benefit from very different interventions.

There is thus no one-size-fits-all intervention. The approach of focusing on the person rather than the label is known as precision psychiatry. This customised approach seeks to target the specific symptoms experienced by the individual. This resonates with Hippocrates' understanding that it's far more important to know what person the disease has than what disease the person has. In order for this to happen, the assessment must be executed and reported in a manner that will highlight the child's strengths and weaknesses in behavioural and cognitive functioning. This information will serve as a basis for intervention by all professionals that interact with the child. School placement will be informed and the practitioner will be in a better position to assist with formulating an Individual Support Plan (ISP) for all multidisciplinary health care professionals involved in establishing a treatment plan.

This paper examines the use of the Griffiths III as a measure to determine a child's overall developmental level and to highlight the child's particular strengths and needs. The ultimate goal would be to use the individual's strengths to improve deficits in other areas. In order to understand the process of thinking of this particular child, 
ToM was used to assist professionals working with the child in understanding the cognitive processes that take place in ASD children.

\section{Methods}

The Griffiths III was originally developed by Dr Ruth Griffiths in 1954 and is intended for children from birth to 6 years of age (72 months) (Green et al., 2016). The Griffiths III provides an overall measure of a child's development, as well as an individual profile of strengths and needs across five subscales, namely (A) Foundations of Learning, (B) Language and Communication, (C) Eye and Hand Coordination, (D) PersonalSocial-Emotional and (E) Gross Motor domains. While JJ's chronological age (6 years 4 months/76 months) technically places him outside the age range for the use of the Griffiths III which caters for children from birth to 5 years 11 months, it was utilised as he presents with atypical patterns of functioning that were expected to fall within the developmental range of the Griffiths III. The Griffiths III is instrumental in determining whether a child is developing age appropriately or whether a general or specific developmental delay is indicated. Identification of specific difficulties could be linked to a specific developmental or learning disorder (The Association for Research in Infant and Child Development, 2018). In particular, the Griffiths III includes a number of different aspects of development, which are likely to be problematic in a child who meets the DSM criteria for ASD. Subscales A, B and D are expected to be the most affected in children on the ASD spectrum.

The Griffiths III thus provides health care practitioners the opportunity to assist in planning developmental and therapeutic interventions and sheds light on future placement and decisions about the management of the child. The Griffiths III is standardized based on a normative sample of 426 children. The sample was developed within the guidelines of continuous norming. On a normed developmental measure, scores are of limited use when they fall below the $50^{\text {th }}$ percentile, and clinicians are challenged as to how to report findings in a manner that will enhance the learning process. For children functioning below a developmental quotient (DQ) of 50 on any of the five developmental domains and for whom a developmental age is not available, developmental age equivalents may be obtained for descriptive purposes using the Griffiths III. A quartile chart with individual items arranged in a tabular format (i.e., 3-month periods) according to difficulty can be used to ascertain at what age this item was passed by a typical child.

Expanding on Griffith's initial belief that the assessment should adapt to the child and not the child to the assessment, quartile charts have been developed which have recently supplemented the developmental measure in order to derive a better understanding of atypical children when their scores fall below the $50^{\text {th }}$ percentile. These charts can assist by indicating at what age a typical child would have managed to complete items, as well as identifying items in the domains that have not been achieved. Merely indicating that the individuals' performance falls below the $50^{\text {th }}$ percentile previously made sense to practitioners, but was not of benefit to the parents and educators who had to assist these individuals.

\section{Results}

The results will be reported according to the five subscales of the Griffiths III, as well as accompanying quartile charts, which provides a further explanation of how scores that fall below the $50^{\text {th }}$ percentile can be interpreted. The use of the Griffiths III and accompanying measures together with collateral history assisted with confirming diagnoses.

\subsection{Subscale A}

The Foundation of Learning Subscale (Subscale A) focuses on the approach taken by the child for future educational experience and includes attention and processing speed abilities. In Subscale A the constructs Skills for Learning (which includes attention, curiosity, processing speed), Ways of Thinking, Memory and Play (in terms of the way the child engages with real objects in imaginative ways) are likely to add definition to the assessment of the foundations of learning. This subscale evaluates the child's natural curiosity and ability to learn by recording the earliest indications of arithmetical comprehension, the realization of the simplest practical problems and the child's ability to memorize information. Foundational skills mainly indicate the child's ability to benefit from formal schooling.

JJ's performance on Subscale A falls in the extremely low range and provides a developmental age equivalent of 55 months. This score suggests a developmental quotient of below 50 , which places JJ below the first percentile rank.

\subsection{Subscale B}

The Language and Communication Subscale (Subscale B) refers to the ability to understand the meaning of words and to use language effectively. Subscale B includes an assessment of a child's use of language to 
communicate socially with others as well as certain aspects of cognition such as attention. This subscale provides an opportunity for the study of the growth and development of both receptive and expressive language.

JJ's performance on Subscale B falls in the extremely low range and provides a developmental age equivalent of 50 months. This score suggests a developmental quotient equal to or below 50, which places $\mathrm{JJ}$ below the first percentile rank.

\subsection{Subscale C}

Eye and hand co-ordination subscale (Subscale C) refers to the early development of visual perception, visually directed reaching and object manipulation. It includes the ability to use small muscle groups to engage in activities. This subscale consists of items relating to the handwork and visual ability of the child.

JJ's performance on Subscale C falls in the borderline range and provides a developmental age equivalent of 57 months. This score suggests a developmental quotient of 80-81, which places JJ at the 11th percentile rank. Eye and Hand Co-ordination appears to be a relative strength when compared to other subscales.

\subsection{Subscale D}

The personal-social-emotional domain (Subscale D) refers to the child's self-care skills such as dressing, undressing, and washing hands. Subscale D contains the underlying subconstructs in early social and emotional development: emotional understanding and expression, self-regulation, joint attention and perspective taking, empathy, moral development and theory of mind. The subscale also provides an opportunity to assess social development and explore the child's social interaction, humour and friendships. It furthermore examines the child's emotional development, for example, by looking at the child's emotional understanding and expression, moral reasoning and attachment.

JJ's performance on Subscale D falls in the extremely low range and provides a developmental age equivalent of 53 months. This score suggests a developmental quotient of below 50 , which places JJ below the first percentile. $\mathrm{JJ}$ evidenced difficulty with all the underlying constructs of early social and emotional development.

\subsection{Subscale E}

Gross-motor skills are measured in Subscale E and refer to the child's early development of postural control, gross body coordination, and visual-spatial coordination. In later years, balance, rhythm, motor sequencing, power, and strength are included. This subscale provides an opportunity to observe physical development in young children.

JJ's performance on Subscale E falls in the borderline range and provides a developmental age equivalent of 56 months. This score suggests a developmental quotient of 74 , which places $\mathrm{JJ}$ at the fifth percentile rank. Taken in relation to the totality of the results, JJ's gross motor skills appear to be a relative strength.

\subsection{Overall Performance}

Considering the results on the Griffiths III holistically, JJ's scores fall within the extremely low range with a developmental age equivalent of 54 months. This is 22 months below his current chronological age. JJ's overall score suggests a developmental quotient of below 50, which places him below the first percentile and implies that his general development can be described as poorer than $99 \%$ of children aged 72 months (6 years). JJ's consistently low performance across all five subscales can be reasonably assumed to indicate significant developmental delays and supports concerns about his behavioural and scholastic difficulties.

In order to analyse the patterns regarding JJ's skill deficits on all subscales, coupled with the fact that only two subscales namely $C$ and $E$ were above the 50th percentile, the accompanying quartile resources developed for the Griffiths III were used to analyse item deficits on JJ's subscale scores that fell below the $50^{\text {th }}$ percentile. In the quartile charts, each Griffiths III item has been placed within the year and subscale groupings according to that item's level of difficulty, which was calculated for the standardisation sample of typically developing children. It may be stated that for test items achieved with delay by the child, typically developing children in the Griffiths III standardisation sample achieved such test items in the 3-month period of the year demarcated.

These charts can also be used to analyse the constructs that have not developed in order to personalise JJ's educational support plan. An example of how such a chart can be utilized is illustrated in Figure 1. The circles in Figure 1 indicate the items and accompanying constructs of each subscale that $\mathrm{JJ}$ scored below the $50^{\text {th }}$ percentile, namely $A, B$ and $D$. 


\section{Griffiths III Year Four Items grouped by Quartile level of difficulty}
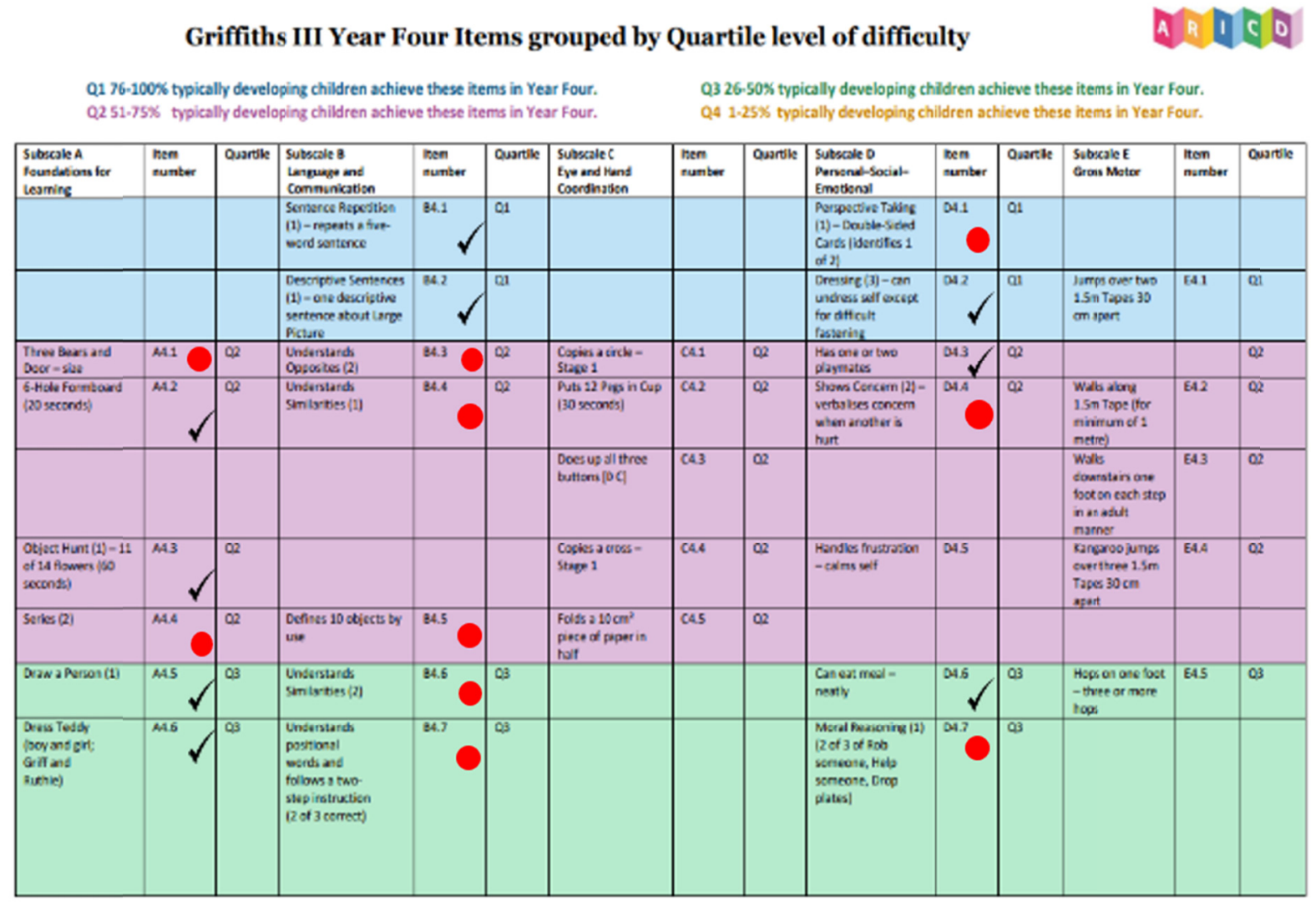

Figure 1. Griffiths III year four items grouped by Quartile level of difficulty

One way to utilise the quartile charts is to examine the functional age across the five subscales. Another use of the charts is to analyse the constructs not achieved, and to use this information to assist with future educational planning.

When analysing the results qualitatively, it becomes evident that the constructs on Foundations of Learning (Subscale A), namely 'Ways of Thinking', 'Verbal Memory' and 'Concentration' are severely compromised. As the score of this subscale fell below the $50^{\text {th }}$ percentile, Figure 1 was used to evaluate the particular constructs that $\mathrm{JJ}$ was unable to achieve. In JJ's case, it is evident that he has difficulties with arithmetical constructs requiring spatial concepts such as 'big' and 'small', as well as incidental memory items and items requiring concentration.

The scores obtained on the Language and Communication Subscale (Subscale B) were the lowest when compared to the other domains. When examining the score on this subscale, it can be seen that it also falls below the $50^{\text {th }}$ percentile and, when consulting the quartile charts, that communicative intent needs encouragement. JJ tends to speak only when spoken to and never engages in spontaneous interactive communication. His expressive language was limited to three-word spontaneous utterances. It was obvious however from his understanding of the instructions used during the assessment that his receptive vocabulary was greater than his expressive language. JJ was able to understand most of the instructions that indicate some receptive language abilities. He was also able to respond to concrete verbs such as "jump", "throw", and other "action-type" verbs, but he had problems with conceptual language concepts.

The quartile charts indicate that constructs not achieved include concepts such as understanding similarities and differences as well as defining objects by use. The quartile charts indicate that $51-75 \%$ of typically developing children in year 4 are able to complete these items successfully, while JJ is 6 years and 4 months. He was also unable to understand positional words that $26-50 \%$ of typically functional children are able to. The percentages published on the resource document are based on the percentage of normal children who passed the item.

The Hand and Eye co-ordination Subscale (Subscale C) score suggest that this domain is a strength as JJ obtained the highest score on this subscale. JJ's performance on this subscale falls in the borderline range and 
provides a developmental age equivalent of 57 months. His score suggests a developmental quotient of 80-81, which places him at the 11 th percentile rank. This implies that $89 \%$ of children aged 6 years old would perform better than him on the Eye and Hand Coordination subscale. Taken in relation to the totality of his results, JJ's Eye and Hand Coordination appear to be a relative strength. However, the execution of some of the items was different from the functioning of most children his age. JJ's strength in this area lies within the fine-motor realm; concretely he was able to place the pegs on the board within the specified time.

When analysing JJ's functioning symbolically on this scale he is able to execute pencil and paper tasks but differently to what was asked of him. He could also produce a relatively detailed drawing of a person; however, he was unable to name the other figures drawn (i.e., his siblings). Additionally, when completing a dot-to-dot diagram of a circle, JJ moved his pencil from one dot to another in order to complete the item in the answer booklet. JJ is unable to use a pair of scissors and also has problems with bilateral co-ordination.

The scores obtained on this subscale do not correlate with the DAP: IQ. JJ's drawing on the DAP: IQ produced an IQ equivalent of 95 , which places him at the $37^{\text {th }}$ percentile. His performance can be qualitatively described as average. Most notably, his drawing suggests impulsivity, immaturity, poor integrative capacity, emotional instability, and aggressive acting out behaviour. Since he scored above the $50^{\text {th }}$ percentile on this subscale, the quartile charts were not needed to provide additional information. Qualitative information, such as JJ's reluctance to interact with the playdough, is one of the observations that could be used to guide the development of an independent support programme in this area of functioning.

The Personal-Social-Emotional Subscale (Subscale D) score was the second lowest and fell below the $50^{\text {th }}$ percentile. On a personal level JJ was able to respond to his name, knew his gender, was toilet trained and could partake in basic self-care tasks, However, he displayed poor eye contact and did not refer to the words "I", "me" and "mine" spontaneously. JJ's parents confirmed this behaviour. One of the problems evidenced on this subscale was JJ's understanding of "perspective-taking" constructs. In Figure 1 the quartile chart D4.1 indicates that $76-100 \%$ of typically developing children at age 4 are able to complete this item.

On a social level $\mathrm{JJ}$ is able to engage in parallel play, but he does not understand any type of rules needed for interactive play and socialisation. The construct that measures D4.4 'Shows Concern' (2) was problematic; when resorting to the quartile charts to understand JJ's functioning this indicates that $51-75 \%$ of typical developing children of 4 years of age pass this item. Emotionally, JJ has an inability to recognise and express thoughts and feelings about himself and others; consequenally he is unable to benefit from group learning that is interactive in nature. Moral reasoning constructs assessed on the measure remain problematic (see Figure 1, D4.7) and when the quartile charts are consulted, they indicate that this construct is achieved by $26-50 \%$ of 4 -year-old typically functioning children.

On the Gross Motor Subscale (Subscale E) JJ was able to perform basic gross motor and visual-spatial coordination activities, such as throwing a tennis ball, kangaroo jumps and walking up and down stairs. However, JJ presented with difficulties with bilateral co-ordination and rhythm such as crossing feet when seated, sequential activities such as hop-jump-hop, basic balancing (walking forward on a tape), and marching to a rhythm dictated by a tambourine.

\section{Discussion}

The Griffiths Developmental Scales assessment indicates JJ's current level of developmental functioning and provides vital additional information to other forms of assessment, which can be used to expand his diagnosis. Previously normed developmental measure scores are of limited use when they fall below the $50^{\text {th }}$ percentile, and clinicians are challenged as to how to report findings in a manner that will enhance the learning process.

As JJ meets the criteria for ASD, his way of thinking needs to be taken into account as this is critical inunderstanding the thinking patterns of young children with ASD. For the purposes of this article, Theory of Mind (ToM) is suggested as it best explains the present case study. This theory encapsulates the thinking of autistic children succinctly, and in a manner that details many of the thought processes of an autistic child, thus guiding tuition.

According to Bogdashina (2005), ToM appears to occur spontaneously in childhood. However, children with autism have impairments in ToM and, in extreme cases, some autistic children may not have the concept of mind at all. ToM deficits affect daily social and academic life in an individual with ASD. However according to Tager-Flusberg (2007) social communication impairments cannot be explained on the basis of ToM impairment, although ToM is a critical factor in the sociocognitive development of children. Baron-Cohen (2009) extended the ToM deficit hypothesis and suggested that sociocommunicative deficits in ASD derive from a delay in the 
development of the empathising system, whereas their intact abilities in systemising (i.e., the ability to construct and analyse systems) may explain the non-social aspects of ASD such as their repetitive behaviours and narrow interests.

Hartly and Branthwalte (2000) state that ToM does not refer to one specific skill, but relates to a series of unfolding level of ten skills. These skills are: (1) the appearance-reality distinction, (2) first-order false belief tests, (3) "seeing leads to knowing", (4) recognising mental state words, (5) using these mental state words in spontaneous speech, (6) spontaneous pretend play, (7) understanding causes of emotions, (8) inferring from gaze direction, (9) deception, and lastly (10) second-order false beliefs. These skills will be briefly discussed using the results obtained from JJ's assessment.

\subsection{Appearance-Reality Distinction}

The appearance-reality distinction is one of the unfolding skills described in ToM. From about the age of 4 years old, typically developing children are able to distinguish between appearance and reality. This can be understood as talking about objects that may have misleading identities (Hartly \& Branthwalte, 2000). For example, a child is presented with an object that is different from its original appearance such as a sponge looking like a rock (Hamilton, Hoogenhout, \& Malcom-Smith, 2016). Children with autism generally do not capture this dual identity; instead, they indicate the object is really a sponge or a rock (Hartly \& Branthwalte, 2000). This was the case with JJ, who was unable to understand "Ways of Thinking" constructs on the Griffiths III and this, combined with limited language abilities, precluded him from understanding conceptual aspects of thinking.

\subsection{First-Order False Belief}

Another skill is that of First-order false belief which is a hallmark task coined by Baron-Cohen, Leslie and Frith (1985). It requires a child to distinguish between the world as it really is and the way it may be represented (incorrectly) in the mind of another. This task measures a child's understanding of the fact that people can have different thoughts about the same situation. Children with ASD have difficulty in shifting their perspectives to what other people might be thinking. A minority of ASD children do pass first-order false belief tests (Hartly \& Branthwalte, 2000). However, individuals who have better language skills are more likely to succeed at this task. $\mathrm{JJ}$ currently uses three-word utterances mainly to express his biological needs and is unable to understand or use conceptual language concepts.

Various studies have found causal relationships between language and ToM development, (Astington \& Jenkins, 1999; Steele, Joseph, \& Tager-Flusberg, 2003). ASD children can often pass false-belief tasks, but their success is dependent on their language skills; yet even the majority of ASD children are unable to act spontaneously according to that knowledge (Senju, 2012). Studies have also shown causal relationships between executive functions and ToM in both typical and atypical children (Pellicano, 2010). It is thus unsurprising that children with ASD, who have difficulties in executive function and cognitive shifting, also show difficulties in predicting false beliefs. Peterson, Garnett, Kelly and Attwood (2009) found that ASD children who passed the false-belief tasks exhibited fewer everyday social and conversational difficulties. JJ was unable to pass both first and second order false belief tests on the Griffiths III indicating limited thoughts about similar situations.

\section{3 "Seeing Leads to Knowing"}

Seeing leads to knowing is an understanding of where knowledge comes from in order for children to work out who knows what and who does now know, and is the foundation of the false belief tests. This reinforces appropriate communication such as telling people what they do not know. For example, in a story of two characters, whereby one looks into a box and another touches a box, children can work out that it is only the one that looked in the box who knows what is in the box, but children with autism perform mainly at chance on this test (Hartly \& Branthwalte, 2000). JJ's thought processes indicate that he cannot predict future actions from prior knowledge, therefore he is unable to generalise knowledge learned. The item assessing this concept indicates that JJ also failed the perspective taking test D5.3 in Subscale D of the Griffiths Scales.

\subsection{Recognising Mental State Words and Using These Words}

Another emerging skill necessary to develop a normal ToM is recognising mental state words such as think, know, dream, pretend, imagine and wish. These are easily distinguishable from other non-mental verbs such as jump, eat or move. JJ was able to understand concrete verbs such as jump and kick both receptively and expressively. Thus, he was able to follow the instructions on Subscale E. However, he had no comprehension of conceptual verbs such as wishing and thinking. He also did not understand rules for social interaction resulting in limited interactive play with others. 


\subsection{Spontaneous Pretend Play}

Spontaneous pretend play is another unfolding skill necessary for healthy development. Symbolic play is relevant because it requires children to decouple the primary representation of an object from its pretend representation. For example, a banana cannot be a banana if one pretends it's a telephone (Leslie, 1987). Children with ASD have such marked impairments in symbolic and pretend play that these deficits become part of the diagnostic criteria for autistic disorders in the Diagnostic and Statistical Manual of Mental Disorders, Fourth Edition (American Psychiatric Association, 1994), and these criteria remained in the subsequent edition. Lam and Yueng (2011) found that the symbolic play of a small group of pre-schoolers with ASD had difficulty mentalising others' perspectives and this was the root of their difficulties in pretend and symbolic play. There is thus a low rate of both interactive and fantasy play in children with autism. This might be because children with autism fail to reflect their own imagination (Hartly \& Branthwalte, 2000).

JJ's parents report that he tends to isolate himself in his room and that he resorts to pencil and paper drawing tasks rather than fantasy play. He also displays limited reciprocity during play, preferring to engage in senseless games such as banging a hammer on the ground until the activity is stopped by another person. Dangerous behaviours such as jumping on moving vehicles are also evident.

\subsection{Understanding Causes of Emotions}

Understanding causes of emotions refers to the fact that emotions can be caused by physical events such as falling and then crying or that it can refer to mental states such as one's desires. Children with autism have difficulty in understanding the causes of emotions (Hartly \& Branthwalte, 2000). The CARS2 indicates that JJ is relatively withdrawn, has flat affect, and has difficulty controlling his intense anger and frustration. He was unable to regulate emotional experiences and he lacked an understanding of emotional expression, emotional understanding and moral reasoning (see these items on the quartile chart: D4.1; D4.4; D4.7.)

\subsection{Inferring from Gaze Direction}

Inferring from gaze direction is another unfolding skill. Typically developing children can understand when someone is thinking about something else such as a gaze directed upwards or away. In contrast, children with autism are relatively blind to such information. Eye gaze is a salient social cue that plays an important role in social interaction and communication, Gaze perception activates a network of brain regions including the posterior temporal sulcus and amygdala, which are central to biological motion and social cognition (Baron-Cohen, 1985). Gaze activates communicative intent. JJ did not make eye contact directly during any part of the assessment and he had a habit of looking at things from an angle or from the corner of his eye. He was unable to read non-verbal gestures and he mainly interacted in his own world. The items in the Griffiths III relating to eye gaze are in Year one so were not tested during JJ's assessment.

\subsection{Deception}

Deception is a further sociocognitive milestone and is the ability to deceive and lie actively, as a distinct ability from recognising lies (Baron-Cohen, 2009). What happens regarding this ability in ASD is that one intentionally instils a false belief in the minds of others and this is considered an important manifestation of ToM (Li, Kelly, Evans, \& Lee, 2011) Studies have examined this task in the context of competition and found that ASD children show difficulty in deceiving. Deception can be explained as trying to make someone believe that something is true, when in fact it is false. Information provided by the CARS2 and Subscale D of the Griffiths III substantiate this aspect. JJ was unable to understand the moral reasoning constructs in the test battery. The results on the CARS-2 indicate that JJ's social emotional understanding is moderately impaired when relating to people. Both JJ's verbal and non-verbal communication are severely impaired, thus further limiting his understanding of deception.

\subsection{Second-Order False Belief Skills}

Second-order false belief skills forms part of ToM and involves considering embedded mental states such as one person's thoughts about another person's thoughts. Children with autism tend to fail second-order false belief tests, but those who pass these tests still display social and communication impairments (Hartly \& Branthwalte, 2000). JJ's limited language, together with his way of thinking, precluded him from understanding this concept resulting in an inability to complete this item.

\subsection{Theory of Mind Interventions}

ToM interventions: These can be divided into specific interventions, general interventions and multimodal interventions. The most important issue in ToM training is the assessment of whether training generalises into 
other tasks, and more importantly into real life. General findings have demonstrated that improvement in ToM skills following specific interventions does not appear to correspond with improved social capabilities (Hadwin, Baron-Cohen, Howlin, \& Hill, 1996). Specific interventions are based on teaching internal, subjective mental representations in oneself and others, which may include role-playing, picturing, thought bubbles and perspective taking (Gould, Tarbox, O’Hara, Noone, \& Bergstrom, 2011; Paynter \& Peterson, 2013). It is important to note that not all skills learned during these interventions can be generalised.

General interventions that incorporate ToM principles and include other social skills seem to be more likely to generalise to other settings (Begeer, Malle, Nieuwland, \& Keysar, 2010). Parents also reported that social skills are more likely to generalise to other settings.

Multimodal Interventions are more sophisticated and, combined with sociocognitive training, are aimed at augmenting the holistic functioning of the child (Bauminger, 2007). This type of training has been found to enhance target skills, but generalisation to other skills have been found to be minimal.

All these principles need to be borne in mind when dealing with ASD children. JJ's cognitive, social and emotional aspects of functioning need multimodal intervention. Cognitively, his communication needs to be remediated by the educator and speech therapist. His unusual behaviour patterns such as running into traffic need to be contained by placing him in special schooling that offers constant care. Interaction with others needs to be encouraged within the school setting. His relative strengths such as gross and fine motor coordination need to be used as rewarding activities when developing a program for him.

\section{Conclusion}

Assessment using the Griffiths III has highlighted JJ's specific difficulties in a number of areas and clarified the nature of his learning difficulties where he is functioning below the $50^{\text {th }}$ percentile. Autism is a complex, disabling disorder that is physically and emotionally demanding on both the patient and their family. The delayed diagnosis of ASD in children imposes negative impact on the outcome and developmental learning of these children, and studies have shown that early diagnosis and consequential early intervention result in better outcomes for the young child. Therefore, it is critical to highlight the importance of providing better diagnostic and intervention facilities and learning strategies to ASD children.

From the above case study, it can be concluded that there are no single role players in the diagnosis, assessment and treatment of children with ASD, thus making this process multi-disciplinary in nature. The assessment must be able to inform parents and educators in a manner that will guide them as to how to use diagnostic results and assessment effectively in order to develop a meaningful support programme for the child assessed. In order for this to happen the assessment must be executed and reported in a manner that will highlight the child's strengths and weaknesses in behavioural and cognitive functioning. This information will serve as a basis for intervention by all professionals that interact with the child.

In the present case study, the assessment tool of choice to determine JJ's cognitive level was the Griffiths III, together with the quartile charts that have recently supplemented the developmental measure in order to derive a better understanding of atypical children when their scores fall below the $50^{\text {th }}$ percentile. The aims of these additional charts are to assist professionals using the Griffiths III to be able to make greater meaning of scores falling below the $50^{\text {th }}$ percentile, as well as to examine the constructs the child is unable to achieve. These charts can assist by indicting at what age a typical child would have managed to complete items, as well as identifying items in the domains that have not been achieved. By using the results creatively, both the constructs evaluated on the Griffiths III, supplemented by the quartile charts, can assist in planning the way forward educationally for a young autistic child. Furthermore, in order to understand the process of thinking of this particular child, ToM was used to assist professionals working with the child in understanding the cognitive processes that take place in ASD children.

It can be concluded that the Griffiths III, together with other measures, is an appropriate assessment tool to assist with the diagnosis and assessment of atypical children, especially autism. Appropriate placement and appropriate support for both parents and children can then follow. Hoerver, the present research consists of a single case study, thus not allowing generalisation of findings. It needs to be acknowledged that multiple case studies may provide further indepth information that would guide professionals in the use of this measure for evaluating atypical children.

\section{Acknowledgements}

We would like to extent our gratitude to the Assosiation for Research in Infant and Child Development for their funding and input and for making this article possible. 


\section{References}

American Psychiatric Association. (1994). Diagnostic and statistical manual of mental disorders (4th ed.). Arlington, VA: American Psychiatric Association.

American Psychiatric Association. (2013). Diagnostic and statistical manual of mental disorders (5th ed.). Arlington, VA: America Psychiatric Association. https://doi.org/10.1176/appi.books.9780890425596

Astington, J., \& Jenkins, J. (1999). A longitudinal study of the relation between language and theory of mind development. Developmental Psychology, 35, 1311-1320. https://doi.org/10.1037//0012-1649.35.5.1311

Baron-Cohen, S, (1995). Mind blindness: An essay on autism and theory of mind. Cambridge, MA, US: The MIT Press. https://doi.org/10.7551/mitpress/4635.001.0001

Baron-Cohen, S. (2009). Autism: The empathizing-systemizing theory (E-S theory). Annals of the New York Academy of Sciences, 1156, 68-80. https://doi.org/10.1111/j.1749-6632.2009.04467.x

Baron-Cohen, S., Leslie, A. M., \& Frith, U. (1985). Does the autistic child have a "theory of mind"? Cognition, 21(1), 37-46. https://doi.org/10.1016/0010-0277(85)90022-8

Bauminger, N. (2007). Individual social multi-modal intervention for HF-ASD. Journal of Autism and Developmental Disorders, 37, 1593-1604. https://doi.org/10.1007/s10803-006-0245-4

Begeer, S., Malle, B. F., Nieuwland, M. S., \& Keysar, B. (2010). Using theory of mind to represent and take part in social interactions. Comparing high functioning autism and typically developing controls. European Journal of Developmental Psychology, 7, 104-122. https://doi.org/10.1080/17405620903024263

Bogdashina, O. (2005). Theory of mind and the triads of perspectives on autism and Aspergers syndrome: A view from the bridge. London: Jessica Kingsley.

Conners, C. K. (2008). Conners 3 - Parent and Teacher Surveys, Long Form (Conners 3) (Conners 3rd ed. manual). Toronto, Ontario, Canada: Multi-Health System.

Draw-a-Person Intellectual Ability Test for Children (DAP: IQ). (1963). Goodenough-Harris Drawing Test.

Gould, E., Tarbox, J., O’Hara, D., Noone, S., \& Bergstrom, R. (2011). Teaching children with autism a basic component skill of perspective taking. Behavior Intervention, 26, 50-66. https://doi.org/10.1002/bin.320

Green, E., Stroud, L., Bloomfield, S., Cronje, J., Foxcroft, C., Hurter, K. et al. (2016). The Griffiths Scales of Childhood Development (3rd ed.). Oxford, UK: Hogrefe Ltd.

Hadwin, J. A., Baron-Cohen, S., Howlin, P., \& Hill, K. (1966). Can we teach children with autism to understand emotions, belief, or pretense? Development and Psychopathology, 8, 345-365. https://doi.org/10.1017/S0954579400007136

Hamilton, K., Hoogenhout, M., \& Malcom-Smith, S. (2016). Neurocognitive considerations when assessing Theory of Mind in Autism Spectrum Disorder. Journal of Child and Adolescent Mental Health, 28(3), 233241. https://doi.org/10.2989/17280583.2016.1268141

Hartly, J., \& Branthwalte, A. (2000). The Applied Psychologist (2nd ed.). Buckingham, U.K.: Open University Press.

Lam, Y. G., \& Yueng, S. S. (2011). Cognitive deficits and symbolic play in pre-schoolers with autism. Research in Autistic Spectrum Disorders, 6, 560-564. https://doi.org/10.1016/j.rasd.2011.07.017

Leslie, A. M. (1987). Pretense and representation: The origin of "theory of mind." Psychological Review, 94, 412-426. https://doi.org/10.1037/0033-295X.94.4.412

Li, A. S., Kelly, E. A., Evans, A. D., \& Lee, K. (2011). Exploring the ability to deceive in children with autism spectrum disorders. Journal of Autism and Developmental Disorders, 41, 185-195. https://doi.org/10.1007/s10803-010-1045-4

Paynter, J., \& Peterson, C. C. (2013). Further evidence of benefits of thought-bubble training for theory of mind development in children with autistic spectrum disorders. Research in Autism Spectrum Disorders, 7, 344 348. https://doi.org/10.1016/j.rasd.2012.10.001

Pellicano, E. (2010). Individual differences in executive function and central coherence predict developmental changes in theory of mind in autism. Developmental Psychology, 46(2), 530. https://doi.org/10.1037/a0018287

Peterson, C. C., Garnett, M., Kelly, A., \& Attwood, T. (2009). Everyday social and conversation applications of 
theory-of-mind understanding by children with autism-spectrum disorders or typical development. European Child \& Adolescent Psychiatry, 18(2), 105-115. https://doi.org/10.1007/s00787-008-0711-y

Schopler, E., Van Bourgondien, M. E., Wellman, G. J., \& Love, S. R. (2010). The Childhood Autism Rating Scale (2nd ed., CARS2). Northumberland, United Kingdom: Ann Arbour.

Senju, A. (2012). Spontaneous theory of mind and its absence in autism spectrum disorders. The Neuroscientist, 18(2), 108-113. https://doi.org/10.1177/1073858410397208

Steele, S., Joseph, R. M., \& Tager-Flusberg, H. (2003). Developmental change in theory of mind abilities in children with autism. Journal of Autism and Developmental Disorders, 33, 461-467. https://doi.org/10.1023/A:1025075115100

Tager-Flusberg, H. (2007). Evaluating the theory-of-mind hypothesis of autism. Current Directions in Psychological Science, 16, 311-316. https://doi.org/10.1111/j.1467-8721.2007.00527.x

The Association for Research in Infant and Child Development. (2018). Griffiths III Year Four Items grouped by Quartile level of difficulty. Retrieved from https://www.aricd.ac.uk/wpcontent/uploads/2018/10/Griffiths-III-Year-Four-Items-grouped-by-Quartile-lev el-of-difficulty.pdf

\section{Copyrights}

Copyright for this article is retained by the author, with first publication rights granted to the journal.

This is an open-access article distributed under the terms and conditions of the Creative Commons Attribution license (http://creativecommons.org/licenses/by/4.0/). 